NOTA CIENTÍFICA

\title{
Dieta de la lagartija de las playas Microlophus peruvianus (Reptilia: Tropiduridae) en la playa Santo Domingo, Ica, Perú
}

1 Facultad de Ciencias Biológicas, Universidad Nacional de Mayor de San Marcos, Apartado 110058, Lima 11, Perú.

2 Departamento de Herpetología. Museo de Historia Natural. Universidad Nacional de Mayor de San Marcos. Email José Pérez perezz_jm@yahoo.com.br

3 Departamento de Ecologia. Instituto de Biologia. Universidade do Estado do Rio de Janeiro (UERJ). Rio de Janeiro, Brasil.

4 Laboratorio de Estudios en Biodiversidad. Departamento de Biodiversidad. Departamento de
Ciencias Biológicas y Fisiológicas. Facultad de Ciencias y Filosofía. Universidad Peruana Cayetano Heredia (UPCH).

Presentado: 30/09/2008

Aceptado: $16 / 10 / 2008$

Publicado online: 26/02/200

\section{Diet of the beach lizard Microlophus peruvianus (Reptilia : Tropiduridae) in the Santo Domingo beach, Ica, Peru}

Eliana Quispitúpac ${ }^{1}$ y José Pérez Z. 2,3,4

\begin{abstract}
Resumen
La lagartija de las playas Microlophus peruvianus es uno de los saurios más conspicuos del Desierto Costero peruano, sin embargo, existe muy poca información sobre su dieta. Se reporta información de la dieta de $M$. peruvianus en la playa Santo Domingo, Ica, que evidencia importantes diferencias entre los principales item alimentarios de juveniles y adultos, que podrían estar reduciendo la competencia intraespecífica.

Palabras clave: dieta, Microlophus peruvianus, Paracas, Ica

\section{Abstract}

Even the beach lizard Microlophus peruvianus is one of the most conspicuous saurian species in the Peruvian desert, however there is almost none information about its diet. Here we reported important evident about the diet differences of both M. peruvianus adult and juvenile in Santo Domingo beach, Ica, which could be reduced the intraspecific competition between them.
\end{abstract}

Keywords: diet, Microlophus peruvianus, Paracas, Ica
La lagartija de las playas Microlophus (=Tropidurus) peruvianus Lesson 1826, es una de las especies más conspicuas del Desierto Costero peruano (Dixon y Wright 1975; Pefaur y Lopez-Tejeda 1983; Pérez y Balta 2007), y su amplia distribución abarca casi todo el litoral peruano desde Piura hasta Arequipa (Dixon y Wright 1975; Carrillo e Icochea 1995, Zeballos et al. 2004). Sin embargo, existe muy poca información sobre su ecología. Se conoce únicamente información limitada de su dieta (Péfaur y López-Tejeda 1983; Pérez y Jhancke 1998; Pérez 2005a; Pérez y Balta 2007) y termorregulación (Huey 1974; Catenazzi et al. 2005).

El objetivo de esta investigación fue evaluar la dieta de $M$. peruvianus en la playa Santa Domingo y analizar las probables diferencias entre juveniles y adultos de esta especie.

El área de estudio fue la playa Santo Domingo, en el límite norte de la Reserva Nacional de Paracas (RNP) (765'9,0"W; $\left.13^{\circ} 51^{\prime} 23,4 " S\right)$, Departamento de Ica, Perú. Esta playa se caracteriza por presentar una franja de dunas paralela a la orilla, en cuya zona intermareal frecuentemente se acumulan algas marinas (Ulva sp.). Estas dunas presentan una altura que varia entre los 1-4 metros. La vegetación en esta playa esta limitada a plantas xerófitas sobre estas dunas, principalmente Sessuvium portulacastrum. En esta playa se han registrado individuos juveniles de las lagartijas Microlophus theresiae y $M$. thoracicus icae (Pérez y Balta 2007), y son frecuentes aves migratorias como playeros (Calidris alba) y chorlos (Charadrius sp.). En alrededores de la zona existen varias edificaciones y es frecuente el tránsito de personas.

La evaluación en campo se realizó entre los meses de abril y mayo de 1996. La captura de lagartijas fue realizada manualmente entre 10:00 -15:00 h. Se eligió este periodo del día para reducir la probabilidad de registrar lagartijas con estómagos vacíos o contenido estomacal muy digerido. Para cada lagartija capturada se registraron datos de longitud hocico-cloaca (SVL) con un calibrador vernier (precisión de $0,1 \mathrm{~mm}$ ), y peso corporal, con una balanza de campo (presión $0,1 \mathrm{~g}$ ). Cada individuo colectado fue identificado como juvenil o adulto, basados en características del patrón de coloración que los diferencian
(Dixon y Wrigth 1975), y de acuerdo a los datos de peso y longitud corporal obtenidos. Todos los individuos colectados fueron preservados siguiendo técnicas estándares en herpetología. El material colectado fue depositado en el Departamento de Herpetología del Museo de Historia Natural de la Universidad Nacional Mayor de San Marcos.

El análisis del contenido estomacal fue realizado en el laboratorio de Entomología de la Universidad Nacional Mayor de San Marcos, con la ayuda de un microscopio estereoscópico. Los item consumidos fueron identificados hasta el nivel de Orden.

Calculamos la amplitud de la dieta (nicho trófico) de $M$. peruvianus mediante el índice de Simpson (Krebs, 1999):

$$
\mathrm{B}_{i j}=1 / \sum\left(p_{i}^{2}\right)
$$

donde, $p_{i}=$ proporción del item $i$ en la dieta.

Se colectaron 11 individuos de $M$. peruvianus (7 juveniles y 4 adultos). Todos los individuos analizados presentaron item en sus estómagos. La dieta de $M$. peruvianus estuvo representada en términos numéricos principalmente por anfípodos $(55,8 \%)$ y coleópteros $(17,8 \%)$ (Tabla 1). Los item más frecuentes en los estómagos analizados fueron los coleópteros (90,9\%), seguidos de anfípodos y larvas de insectos (ambos 72,7\%) (Tabla 1). La amplitud en la dieta de $M$. peruvianus fue $\mathrm{B}_{i j}=2,77$.

En el caso de los juveniles, se observó un mayor número de individuos de coleópteros, representando el 34.2\% de su dieta, seguido por larvas de insectos (19,3\%) y dípteros $(15,5 \%)$ (Tabla 1).

Los adultos presentaron una marcada tendencia por los anfípodos del género Orchestia sp., que representó el 93,84\% de su dieta (Tabla 1). Cabe destacar que los anfípodos fueron el item principal en todos los individuos adultos evaluados. La amplitud en la dieta fue mayor en juveniles $\left(\mathrm{B}_{i j}=4,75\right)$ que en adultos $\left(\mathrm{B}_{i j}=1,13\right)$ (Tabla 1$)$.

Los juveniles de $M$. peruvianus fueron registrados alejados de la orilla del mar, mientras que los adultos, fueron registrados 
Tabla 1. Dieta de juveniles y adultos de Microlophus peruvianus en la playa Santo Domingo, Ica, Perú. Para cada item alimentario se indica el número de individuos, frecuencias y sus respectivos porcentajes (\%)

\begin{tabular}{|c|c|c|c|c|c|c|c|c|}
\hline & \multicolumn{2}{|c|}{ Juveniles } & \multicolumn{2}{|c|}{ Adultos } & \multicolumn{2}{|c|}{ Total } & \multirow[b]{2}{*}{ Frecuencia } & \multirow[b]{2}{*}{$(\%)$} \\
\hline & Número & $(\%)$ & Número & $(\%)$ & Número & $(\%)$ & & \\
\hline Coleoptera & 64 & 34,2 & 7 & 3,32 & 71 & 17,8 & 10 & 90,9 \\
\hline Diptera & 29 & 15,5 & 2 & 0,95 & 31 & 7,8 & 7 & 63,6 \\
\hline Hymenoptera & 21 & 11,2 & 1 & 0,47 & 22 & 5,5 & 7 & 63,6 \\
\hline Hemiptera & 10 & 5,3 & 0 & 0,00 & 10 & 2,5 & 3 & 27,3 \\
\hline Lepidoptera & 1 & 0,5 & 0 & 0,00 & 1 & 0,3 & 1 & 9,1 \\
\hline Larvas de Insectos & 36 & 19,3 & 1 & 0,47 & 37 & 9,3 & 8 & 72,7 \\
\hline Amphipoda & 24 & 12,8 & 198 & 93,84 & 222 & 55,8 & 8 & 72,7 \\
\hline Arachnida & 2 & 1,1 & 2 & 0,95 & 4 & 1,0 & 3 & 27,3 \\
\hline Índice de Simpson & \multicolumn{2}{|c|}{4,75} & \multicolumn{2}{|c|}{1,13} & \multicolumn{2}{|c|}{2,97} & & \\
\hline
\end{tabular}

sobre las dunas y en la orilla del mar. Los juveniles y adultos de Microlophus peruvianus utilizan como refugio las plantas xerófitas de las dunas.

La considerable diversidad en la dieta de $M$. peruvianus observada en este estudio $\left(\mathrm{B}_{i j}=2,77\right)$, también ha sido registrada en otras evaluaciones, donde incluso se han observado mayores valores de diversidad. Por ejemplo, a partir de los datos presentados por Péfaur y López-Tejeda (1983), se calcula una amplitud de $\mathrm{B}_{i j}=5,69$ en la dieta de $M$. peruvianus de las lomas de Matarani en Arequipa, y Pérez y Balta (2007) reportan una amplitud de $\mathrm{B}_{i j}=3,00$ para otras localidades de la RNP. No existen muchos estudios sobre la dieta de especies del mismo género en Perú que nos permitan realizar comparaciones para este género, sin embargo, Pérez (2005b) evaluando la dieta de $M$. tigris en las lomas de Lachay registra una amplitud de $\mathrm{B}_{i j}=4,33$.

Debido a esta considerable amplitud en el nicho trófico, la lagartija de las playas $M$. peruvianus es considerada una especie con dieta de tipo generalista (Péfaur y López-Tejeda 1983; Pérez y Balta 2007) y oportunista (Pérez y Balta 2007). Esta lagartija consume principalmente artrópodos (Péfaur y López-Tejeda 1983; Pérez y Jhancke 1998; Pérez y Balta 2007), también consume material vegetal (Péfaur y López-Tejeda 1983; Pérez y Balta 2007), e inclusive individuos juveniles de su propia especie (Pérez 2005a).

En este estudio se observa una marcada diferencia entre las dietas de juveniles y adultos de M. peruvianus. Ésta diferencia se explicaría principalmente por la división del hábitat registrada en la Playa Santo Domingo. Los adultos predominan en la zona intermareal y los juveniles alejados de la playa. Los anfípodos predominan entre las algas marinas acumuladas en la orilla, lo cual los convierte en el alimento de mayor disponibilidad en este hábitat, explicando su mayor consumo por parte de los adultos de $M$. peruvianus. La especie de invertebrado predominante en la playa Santo Domingo fue el anfípodo semiterrestre Orchestia sp. asociado a la arena húmeda de la orilla de la playa.

Por otro lado, los juveniles fueron observados alejados de la zona intermareal, donde los anfípodos no serían tan abundantes, y por lo tanto, los juveniles consumirían item como coleópteros y larvas de insectos, que serían las presas más frecuentes en las zonas que habitan los juveniles. La gran amplitud o diversidad en la dieta de los juveniles, en comparación con los adultos, evidenciaría una mayor búsqueda alimentos a falta de uno tan abundante como los anfípodos. Estos resultados evidencian el carácter generalista y oportunista de la dieta de $M$. peruvianus.
La marcada diferencia en el uso del hábitat entre juveniles y adultos de $M$. peruvianus ha sido registrada en otras localidades de la Reserva Nacional de Paracas (Pérez y Balta 2007). Estas diferencias en el uso del hábitat estarían reflejando estrategias de división en el uso de recursos para reducir la competencia intraespecífica.

\section{Agradecimientos}

Agradecemos Katya Balta, Jean Matos y Enrique Pinto por su colaboración en el trabajo de campo. Al personal de la Reserva Nacional de Paracas por el apoyo logístico. A Norberta Martínez por la ayuda en la identificación de algunos artrópodos.

\section{Literatura Citada}

Carrillo N. \& J. Icochea. 1995. Lista taxonómica preliminar de los Reptiles vivientes del Perú. Publicaciones del Museo de Historia Natural Universidad Nacional Mayor de San $\operatorname{Marcos}$ (A) 49:1-27.

Catenazzi A.; Carrillo, J. \& M. Donnelly. 2005. Seasonal and Geographic Eurythermy in a Coastal Peruvian Lizard. Copeia 4: 713-723.

Dixon J. \& J. Wright. 1975. A review of the lizards of the iguanid genus Tropidurus in Peru. Contribution in Science of Los Angeles County Museum. 271: 1-39.

Huey R. 1974. Winter thermal ecology of the iguanid lizard Tropidurus peruvianus. Copeia (1):149-155.

Krebs C. 1999. Ecological Methodology. Addison Wesley Longman.

Pefaur J. \& E. López-Tejeda. 1983. Ecological notes on the lizard Tropidurus peruvianus in southern Peru. Journal of Arid Environmnets 6:155-160.

Pérez Z. J. 2005a. Microlophus peruvianus. Cannibalism. Herpetological Review 36 (1): 63.

Pérez Z. J. 2005b. Ecologia de Duas Espécies de Lagartos Simpatricos em uma Formação Vegetal de Lomas no Deserto Costeiro Peruano Central. Dissertação de Mestrado. Universidade do Estado do Rio de Janeiro (UERJ). Rio de Janeiro. Brasil.

Pérez Z. J. \& K. Balta. 2007. Ecología de la comunidad de saurios diurnos de la Reserva Nacional de Paracas. Revista Peruana de Biología 13(3): 169-176.

Pérez J. \& J. Jhancke. 1998. Saurios como consumidores de ectoparásitos de aves guaneras. Boletín del Instituto del Mar de Perú. 81-86.

Zeballos P. Horacio, E. López, L. Villegas, P. Jiménez \& R. Gutiérrez. 2004. Distribución de los reptiles de Arequipa, sur del Perú. Dilloniana 4 (2) 